\title{
Neutral absorber dips in the periodic burster LMXB XB 1323-619 from Suzaku
}

\author{
M. Bałucińska-Church ${ }^{1,2}$, T. Dotani ${ }^{3,4}$, T. Hirotsu ${ }^{3,4}$, and M. J. Church ${ }^{1,2}$ \\ 1 School of Physics and Astronomy, University of Birmingham, Birmingham, B15 2TT, UK \\ e-mail: [mbc;mjc]@star.sr.bham.ac.uk \\ 2 Astronomical Observatory, Jagiellonian University, ul. Orla 171, 30-244 Cracow, Poland \\ 3 Institute of Space \& Astronautical Science, 3-1-1 Yoshinodai, Sagamihara, Kanagawa 229-8510, Japan \\ 4 Department of Physics, Tokyo Institute of Technology, Ohokayama, Meguro, Tokyo, 152-8551, Japan
}

Received 23 October 2008 / Accepted 27 March 2009

ABSTRACT

\begin{abstract}
We present results of an observation with Suzaku of the dipping, periodic bursting low mass X-ray binary XB 1323-619. Using the energy band $0.8-70 \mathrm{keV}$, we show that the source spectrum is well-described as the emission of an extended accretion disk corona, plus a small contribution of blackbody emission from the neutron star. The dip spectrum is well-fitted by the progressive covering model in which the extended ADC is progressively overlapped by the absorbing bulge of low ionization state in the outer accretion disk and that dipping is basically due to photoelectric absorption in the bulge. An energy-independent decrease of flux at high energies $(20-70 \mathrm{keV})$ is shown to be consistent with the level of Thomson scattering expected in the bulge. An absorption feature at $6.67 \mathrm{keV}$ (Fe XXV) is detected in the non-dip spectrum and other possible weak features. In dipping, absorption lines of medium and highly ionized states are seen suggestive of absorption in the ADC but there is no evidence that the lines are stronger than in non-dip. We show that the luminosity of the source has changed substantially since the Exosat observation of 1985, increasing in luminosity between 1985 and 2003, then in 2003-2007 falling to the initial low value. X-ray bursting has again become periodic, which it ceased to be in its highest luminosity state, and we find that the X-ray bursts exhibit both the fast decay and later slow decay characteristic of the rp burning process. We present arguments against the recent proposal that the decrease of continuum flux in the dipping LMXB in general can be explained as absorption in an ionized absorber rather than in the bulge in the outer disk generally accepted to be the site of absorption.
\end{abstract}

Key words. accretion, accretion disks - stars: binaries: close - line: formation - stars: neutron - X-rays: binaries stars: individual: XB 1323-619

\section{Introduction}

XB 1323-619 is a member of the dipping class of Low Mass $\mathrm{X}$-ray Binaries (LMXB), exhibiting reductions in X-ray intensity at the orbital period due to absorption in the bulge of the outer accretion disk (White \& Swank 1982; Walter et al. 1982). It is remarkable as one of the two "clocked" X-ray bursts sources in which bursting remains close to periodic over extended periods of time, the other source being 4U 1826-24 (Galloway et al. 2004). Other sources such as 4 U 1705-44 (Langmeier et al. 1987) have shown bursting close to periodic, but this is not sustained over long periods of time (Cornelisse et al. 2003). The dipping sources display relatively complex spectral evolution in dipping, which provides strong constraints on emission models for LMXB in general since the model must be able to fit the non-dip state and a sequence of spectra formed by selection in intensity bands at different depths of dipping. Work over a period of 15 years provided substantial evidence for a model in which the X-ray emission consists primarily of Comptonized emission from the accretion disk corona (ADC) plus simple blackbody emission from the neutron star (Church et al. 1997, 1998a,b, 2005; Bałucińska-Church et al. 1999, 2000; Smale et al. 2001; Barnard et al. 2001). This work establishes that in the dip sources in general, the dominant Comptonized emission is removed slowly showing that the emission is extended, while the point-like blackbody emission which we associate with the neutron star is rapidly removed, and causes rapid variability in dipping. Measurement of dip ingress times allows the size of the extended emitter to be measured, and this has shown the ADC to reach over a large part of the inner accretion disk, have a radial extent $R$ between 20000 and $700000 \mathrm{~km}$, and is thin, with height $H$ having $H / R \ll 1$ (Church \& Bałucińska-Church 2004). Strong independent support for the extended ADC comes from the Chandra grating results of Schulz et al. (2009) in which emission lines of highly ionizied species were found to originate at radial distances between 20000 and $110000 \mathrm{~km}$ in an extended ADC. Various authors have addressed theoretically the question of the formation, size and properties of an ADC, such as Jimenez-Garate et al. (2002, and references therein) by considering the effects of illumination by the central source.

In the present work we investigate spectral evolution in dipping utilising the broadband capability of Suzaku, the long-term luminosity evolution of the source and the associated strong changes in burst properties. In general, the X-ray burst sources do not have regular bursting. If it is assumed that the mass accretion rate does not vary greatly on timescales of a few hours, regular bursting would be expected, since mass accumulates at a steady rate on the surface of the neutron star and conditions for unstable nuclear burning are achieved. However, the majority of sources have quite irregular bursting and the study of the two "clocked bursters" is clearly of interest in explaining this. The source was observed by us using XMM-Newton 
in 2003, January, and analysis of this observation and comparison with observations previously made with Exosat, ASCA, and three observations with $R X T E$ revealed relatively large systematic changes in burst properties and in the luminosity of XB 1323-619 over the period 1985-2003 (Church et al. 2005). The most striking feature was the changing burst rate, the time between bursts $\Delta t$ decreasing linearly with time from $322 \mathrm{~min}$ in 1985 to $58 \mathrm{~min}$ in 2003. During this period the X-ray luminosity in the band $1-10 \mathrm{keV}$ increased substantially from $1.3 \times 10^{36}$ to $5.4 \times 10^{36} \mathrm{erg} \mathrm{s}^{-1}$. The observed trend if continued would mean that the burst rate would continue to increase, implying that $\Delta t$ would become zero on Jan. 11, 2008. However, analysis of a further observation with RXTE in 2003, September showed a sudden change in the source, the luminosity decreasing and the time between bursts increasing to a mean of $162 \mathrm{~min}$ (Bałucińska-Church et al. 2008).

XB 1323-619 was discovered using Uhuru and Ariel V (Forman et al. 1978; Warwick et al. 1981) and X-ray bursting and dipping found using Exosat by van der Klis et al. (1985). An orbital period of $2.938 \pm 0.020 \mathrm{~h}$ was determined by Bałucińska-Church et al. (1999) in good agreement with the value of 2.932 $0.005 \mathrm{hr}$ from Exosat (Parmar et al. 1989). The source was observed with BeppoSAX in 1997 from which a detailed study of the broadband 1-200 keV spectrum was made (Bałucińska-Church et al. 1999) showing the Comptonized emission to have a relatively high cut-off energy of $44 \mathrm{keV}$. The $X M M$ observation (Church et al. 2005) revealed various line and possible edge features, including $\mathrm{Fe}$ absorption lines at 6.70 and $6.98 \mathrm{keV}$ equivalent to $\mathrm{Fe} \mathrm{XXV} \mathrm{K}_{\alpha}$ and $\mathrm{Fe}$ XXVI $\mathrm{K}_{\alpha}$ and an emission line at $6.82 \mathrm{keV}$. In addition absorption features were seen at $1.46 \mathrm{keV}$ and $4.05 \mathrm{keV}$, possibly $\mathrm{Mg}$ XII and $\mathrm{Ca}$ XX. The origin of lines was discussed and it was concluded that the evidence favoured production in the ADC.

In analysis of the XMM data, Boirin et al. (2005) and Díaz-Trigo (2006) suggested that the dipping in all LMXB dipping sources could be explained in terms of an ionized absorber rather than in the low ionization state bulge in the outer disk generally accepted as the site of absorption. This model would require dipping to be unconnected with the bulge in the outer disk. In the present observation we show that dipping is very well explained as absorption in the bulge plus a small degree of energy-independent electron scattering consistent with the measured column density of the cool absorber. In Sect. 4 we discuss general objections to the ionized absorber model.

\section{Observations and analysis}

We observed XB 1323-619 with Suzaku in 2007, Jan. 9-10 for $34.1 \mathrm{~h}$, this covering 11.7 orbital cycles. Data from both the XIS and HXD instruments were used. Analysis was carried out using the Suzaku-specific Ftools, part of FTOoLS 6.3.1. At ISAS, the XIS raw telemetry files were converted to Fits format using the facility MK1STFITS, and then the raw data files were converted to filtered and calibrated events files using MK2NDFITS. This second stage was carried using the improvements in calibration data of August, 2007 (version 2 processing). The XIS was operated in the normal mode, using the one-quarter window option viewing $1 / 4$ of the $C C D$ and with the $3 \times 3$ and $5 \times 5$ pixel options for pulse height analysis and with $2 \mathrm{~s}$ time resolution. Spaced-row charge injection (SCI) was used to reduce the effects of radiation damage. Data for each of the three detectors XIS0, XIS1 and XIS3 detectors were available, XIS2 having failed prior to this observation. The filtering consisted of the removal of hot and flickering pixels and of selection on event "grade" from the pulse height distribution (keeping grades $0,2,3,4$ and 6 ) to discriminate between X-ray and charged particle events, and also standard screening based on housekeeping data as follows.

This standard screening excluded periods of South Atlantic Anomaly passage plus an elapsed time of 436 seconds after the time of leaving SAA (T_SAA > 436), to select data for elevation above the Earth's limb more than 5 degrees (ELV $>5$ ), for elevation above the sunlit Earth more than 20 degrees (DYE_ELV > $20)$, and for geomagnetic rigidity $(\mathrm{COR}>6)$. Lightcurves were extracted from each XIS detector from the filtered events file using Xselect. The lightcurve obtained after screening revealed only a single burst and 9 dips, whereas a lightcurve made with relaxed screening criteria (below) showed 5 bursts, the last of which was double, and eleven dips, so clearly standard screening removed substantial amounts of data of interest and we investigated the effects of relaxing the selection criteria.

To do this, it was necessary to use the unfiltered events file. Firstly, hot and flickering pixels were removed using the FTOOL CLEANSIS and grade selection was made within Xselect. Inspection of the housekeeping data showed that during the observation, elevation of the source above the Earth's limb was always above $7.5^{\circ}$, i.e. no Earth occultation took place and so there was no need to screen for this. However, we found that when we reduced the daytime elevation selection criterion to DYE_ELV $>10^{\circ}$, more bursts were revealed and we eventually made no selection on this parameter. We then checked the effect of this on spectral data, since X-rays from the bright Earth have a very soft spectrum dominated by $\mathrm{O}$ and $\mathrm{N}$ lines below $1 \mathrm{keV}$. Non-burst spectra with and without screening for DYE_ELV were extracted, but no differences between them could be seen, particularly below $1 \mathrm{keV}$. As the spectrum of XB 1323-619 in the XIS does not extend below $\sim 0.8 \mathrm{keV}$ because of its low Galactic latitude and associated large column density, little effect would be expected.

The geomagnetic rigidity (COR) was also examined. With the standard selection: COR $>6$, a small fraction of data was removed including the first burst at time $\sim 6000 \mathrm{~s}$; see Fig. 1 in which the COR value was 5 . Therefore to include all the bursts we relaxed the selection criterion to $\mathrm{COR}>5$. This may slightly increase the background, however as XB 1323-619 is a bright source in XIS, the overall effect will be negligible. It is clear that our approach of relaxing the selection criteria will not affect the determination from the lightcurve of the burst repetition rate and decay times, and our testing showed that the effect on spectra was negligible.

With the final selections above, the lightcurves from each XIS detector were examined for traces of the nearby pulsar 1SAX J1324.4-6200 (Angelini et al. 1998; Bałucińska-Church et al. 1999) located $17^{\prime}$ from XB 1323-619 which would have contaminated lightcurves and spectra. The observation had been designed with a pointing direction to minimize possible effects. No trace of pulsations could be seen. A lightcurve of XB 1323619 was then extracted using all 3 XIS detectors which were each operated in the mode in which one quarter of the full detector was used consisting of a strip of $1024 \times 256$ pixels. The lightcurve was extracted in the full energy band $0.2-12 \mathrm{keV}$ by specifying an approximately rectangular source region with the source at the centre of size $4.4^{\prime} \times 6.7^{\prime}$; background was extracted using two square regions in the image, each side of the source at a distance of $7^{\prime}$ and $4.4^{\prime}$ square, and adding these. Background subtraction was carried out using LCMATH allowing for the different areas of the source and background regions. Photon pileup is negligible. The background-subtracted lightcurve of the 3 XIS detectors is shown in Fig. 1. 


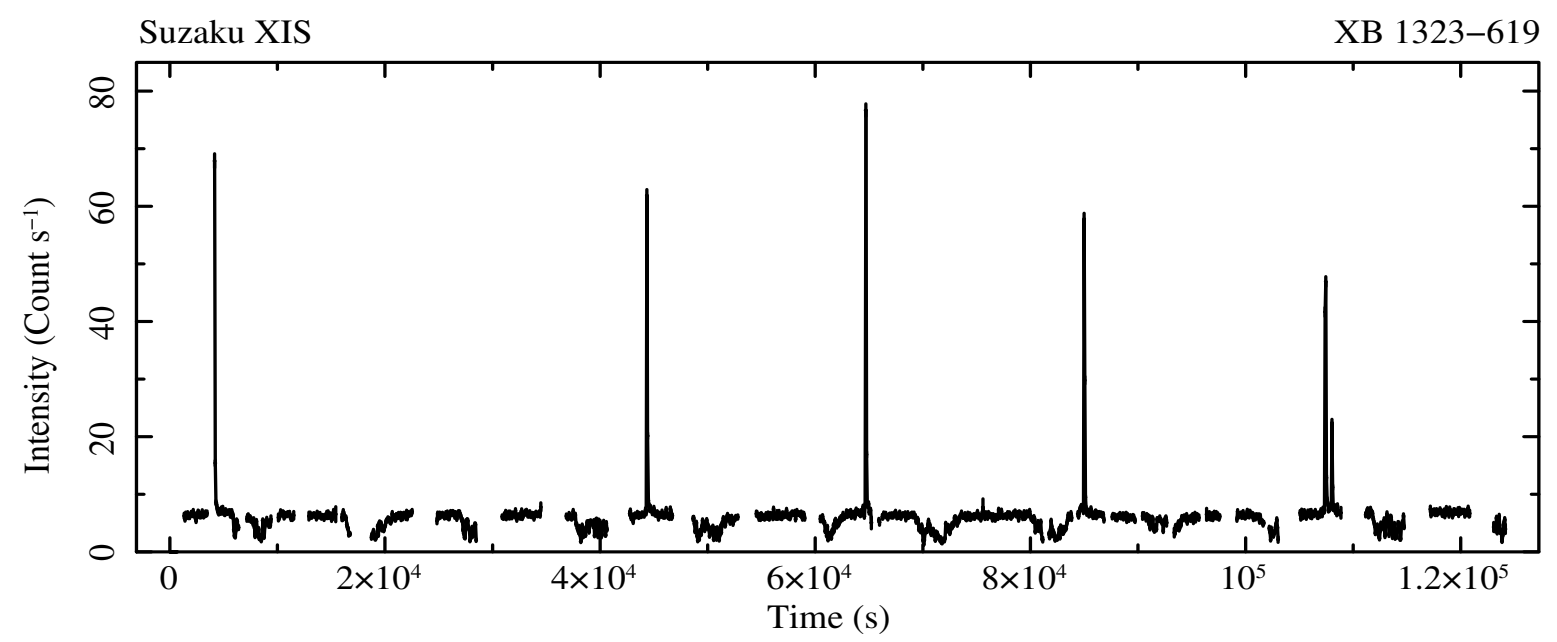

Fig. 1. Background-subtracted lightcurve of the January 2007 observation of XB 1323-619in the energy band 0.2-12 keV with 64 s binning from the XIS detectors XIS0, XIS1 and XIS3.

Exosat, Asca, SAX, RXTE, XMM, Suzaku

XB $1323-619$

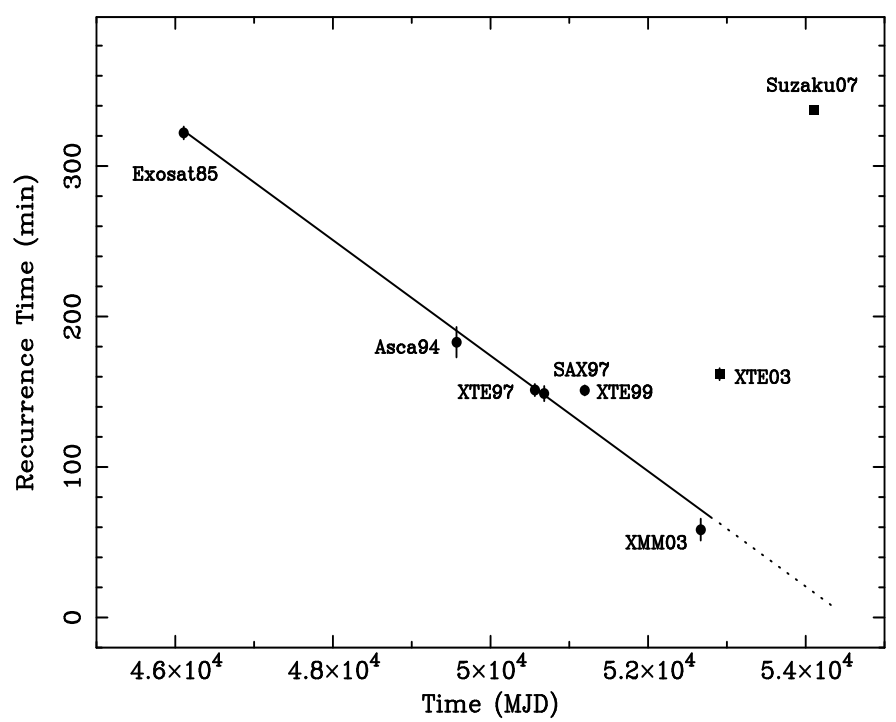

Exosat, Asca, SAX, RXTE, XMM, Suzaku

XB 1323-619

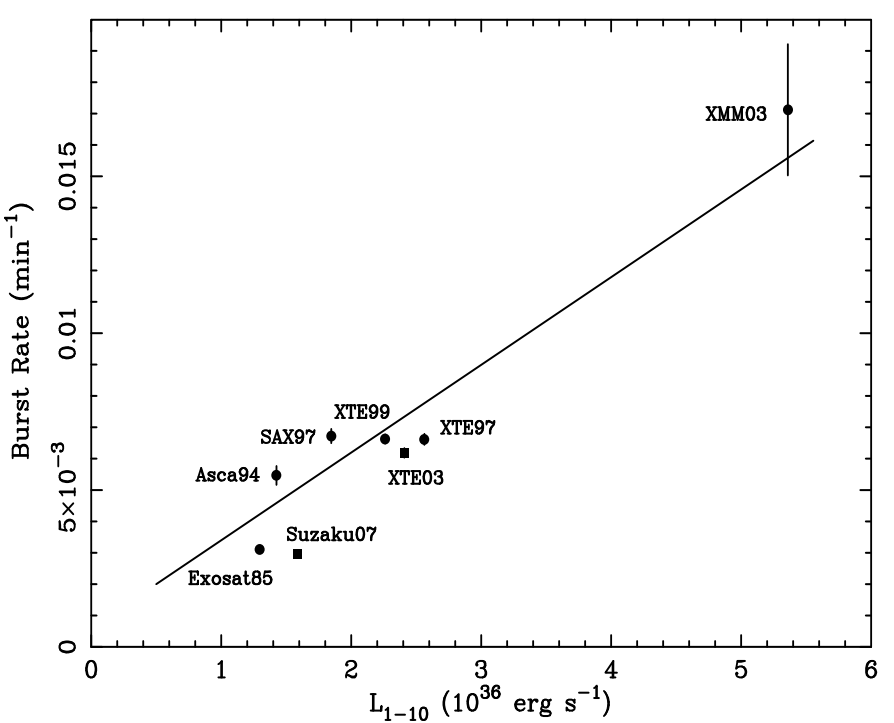

Fig. 2. Left panel: recurrence time $\Delta \mathrm{t}$ of $\mathrm{X}$-ray bursts showing the systematic decrease of $\Delta \mathrm{t}$ from the Exosat observation to the $X M M$ observation followed by the departures from that pattern of the 2003 RXTE and the present observations; right panel: the dependence of burst rate $1 / \Delta \mathrm{t}$ as a function of luminosity. There is an approximately linear relation from the Exosat to the XMM observation, although the 2003 RXTE and Suzaku points have reverted to the positions consistent with the much decreased luminosities.

HXD data were obtained in the form of a cleaned events file in which standard screening was carried out for SAA passage, elevation etc. We analysed the PIN data and found that the source is detected up to $\sim 50 \mathrm{keV}$. Because of this and the weakness of the source, we did not use the high energy GSO data in the band $30-600 \mathrm{keV}$. Non X-ray background in HXD/PIN is evaluated using a background model provided for observers for each observation. The cosmic X-ray background which is about $5 \%$ of the total is included as a model term in spectral fitting using a power law form corresponding to previous determinations of the spectrum. Data were selected via a good time interval file (GTI) ensuring that both source and background data were available. Lightcurves were background-subtracted using the instrumental background, and deadtime corrected using HXFDTCOR. DYE_ELV filtering is not carried out and so does not affect the number of bursts seen; however, COR $>6$ is chosen, so the first burst seen in XIS is removed, but the remaining bursts are seen.

\section{Results}

\subsection{The burst rate}

The lightcurve of Fig. 1 shows 5 bursts, the last of which is double. We first obtain the mean time between bursts $\Delta t$ and thus the burst rate $\left(\Delta t^{-1}\right)$. This was done by examining the XIS0 lightcurve with its full $2 \mathrm{~s}$ resolution, i.e. without $64 \mathrm{~s}$ binning. The times of the peaks of the bursts and separations between the bursts are listed in Table 1. The gap between the 1st and 2nd bursts is consistent with being twice the approximate separation of bursts, and it is almost certain that one burst between these fell in a data gap and so we use half the gap between these bursts. We exclude the double burst from the analysis and using the gaps between the other bursts we find that $\Delta t=20217 \pm 112 \mathrm{~s}$ (with $1 \sigma$ errors), i.e. $\Delta t=5.62 \pm 0.03 \mathrm{~h}$.

Figure 2 (left panel) shows the evolution of burst recurrence time $\Delta t$ with time, i.e. MJD spanning the period 1985 to 
the Suzaku observation. Analysis producing $\Delta t$ is taken from Bałucińska-Church et al. (2008) including values derived for the observations with Exosat, ASCA, RXTE, XMM-Newton and added to it is the point for the present Suzaku observation. It can be seen that the burst rate shows a systematic linear decrease from the Exosat observation to the XMM observation (2003, Jan. 29) as shown by the best-fit solid line. The observations of XTE 2003 (2003, Sep. 25) and the present observation do not follow the trend as marked with the dotted line. It appears that a marked change took place within the source in 2003.

From spectral fitting below using the best fit to the broadband spectrum of the non-burst, non-dip data we obtain a $1-10 \mathrm{keV} \mathrm{lu}$ minosity of $1.47 \times 10^{36} \mathrm{erg} \mathrm{s}^{-1}$, and we next compare the Suzaku data with our previous work on XB 1323-619 in which we obtained the dependence of burst rate on source luminosity from the Exosat observation of 1985 to the RXTE observation of 2003, a span of 18 years (Bałucińska-Church et al. 2008). Burst rate as a function of luminosity is shown in Fig. 2 (right panel) including our analysis of the observations with Exosat, ASCA, RXTE, $X M M$-Newton and the present Suzaku observation. The solid line represents a linear fit to the data up to the 2003 XTE observations. Firstly, it can be seen that the 2003 XTE point follows the linear relation between the luminosity and the burst rate. Moreover, the point for Suzaku is close to the Exosat point, indicating not only that the source has jumped back to its luminosity of 1985, but also that the burst rate is consistent with that luminosity.

\subsection{The burst properties}

We determined the times of the burst peaks, the time between bursts and examined the decays of the bursts and for this, the $64 \mathrm{~s}$ binning of Fig. 1 was not adequate and so we produced a lightcurve from XIS0 with $2 \mathrm{~s}$ binning, this having more than sufficient count statistics. The decay profiles of the bursts were examined, and it was immediately obvious that each burst had a fast decay followed by a much slower decay giving each burst a duration of more than 200 s. This behaviour was typical of the source in previous observations (Bałucińska-Church et al. 2008) and the slow decay indicates nuclear burning by the the rp-process (Wallace \& Woosley 1981; Galloway et al. 2004) was operating. We fitted each burst separately with a function $f(t)=$ $A+N_{1} \times \exp \left[-\left(t-t_{0}\right) / \tau_{1}\right]+N_{2} \times \exp \left[-\left(t-t_{0}\right) / \tau_{2}\right.$ in the vicinity of each burst, i.e. extending $600 \mathrm{~s}$ beyond the burst peak. The function contains two time constants $\tau_{1}$ and $\tau_{2}, t_{0}$ is the burst peak time, $A$ is the persistent emission count rate and $\mathrm{N}_{1}, \mathrm{~N}_{2}$ are normalizations. The results are shown in Table 1, from which we find that the mean exponential decay times are $\tau_{1}=9 \pm 1.8$ and $\tau_{2}=33 \pm 7 \mathrm{~s}$.

The double burst is shown in the table as bursts $5 \mathrm{a}$ and $5 \mathrm{~b}$; it is interesting that the second weaker component $5 \mathrm{~b}$ displayed no evidence for a slow decay.

\subsection{X-ray dipping}

The X-ray dipping was examined by first creating a lightcurve from which all portions of all bursts were excluded to prevent this interfering with the determination of the best-fit orbital period using the dipping. The XIS lightcurve which was a sum of background-subtracted lightcurves from XIS0, XIS1 and XIS3 in the full band $0.2-12.0 \mathrm{keV}$ was searched for periodicities using the PERIOD program which gave a best fit period of 2.926 ${ }_{-0.25}^{+0.38} \mathrm{~h}$. To reduce the uncertainties, a second method was used
Table 1. Measured burst properties.

\begin{tabular}{lrrrr}
\hline \hline Burst No. & $\begin{array}{l}\text { Peak } \\
\text { Time }(\mathrm{s})^{1)}\end{array}$ & $\begin{array}{r}\text { Separation } \\
\Delta \mathrm{t}(\mathrm{s})\end{array}$ & $\begin{array}{r}\tau_{1} \\
(\mathrm{~s})\end{array}$ & $\begin{array}{r}\tau_{2} \\
(\mathrm{~s})\end{array}$ \\
\hline 1 & 4148.0 & $\ldots$ & $6.8_{-1.5}^{+2.3}$ & $26.7_{-2.4}^{+3.6}$ \\
2 & 44358.0 & 40210.0 & $10.4 \pm 2.8$ & $27.9_{-8.9}^{+3.9}$ \\
3 & 64696.0 & 20338.0 & $6.7_{-2.0}^{+2.9}$ & $28.2_{-2.7}^{+4.4}$ \\
4 & 85016.0 & 20320.0 & $11.1 \pm 1.2$ & $46.1_{-12.2}^{+28}$ \\
$5 \mathrm{a}$ & 107420.0 & 22404.0 & $8.6_{-2.7}^{+3.2}$ & $35.5_{-3.0}^{+4.6}$ \\
$5 \mathrm{~b}$ & 108054.0 & 634.0 & $10.8 \pm 0.9$ & $2)$ \\
\hline
\end{tabular}

1) Times relative to MJD 54109.48056. ${ }^{2)}$ No long decay was present in burst $5 b$.

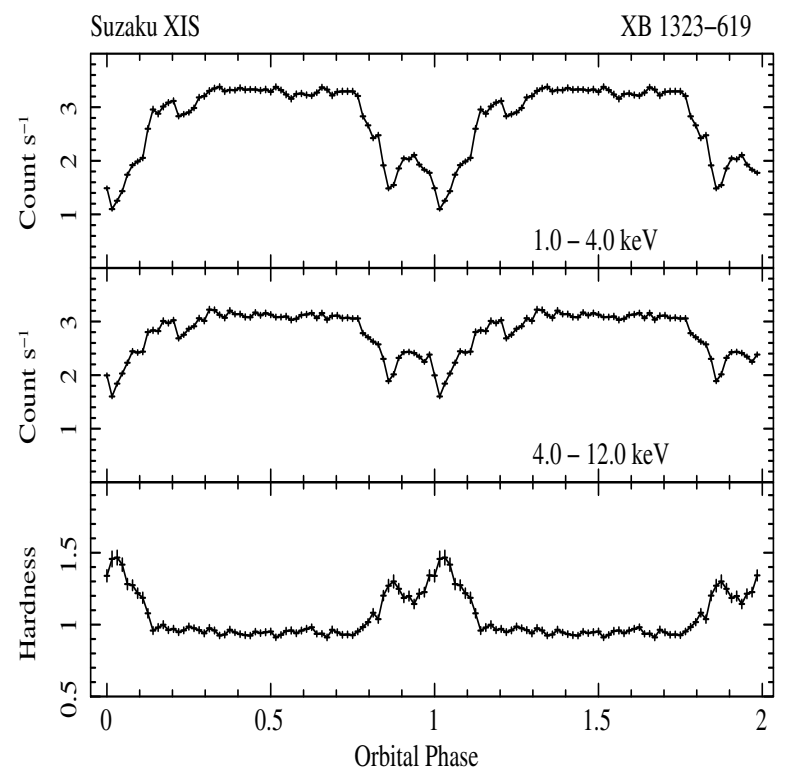

Fig. 3. Background subtracted XIS lightcurves (the sum of XIS0, XIS1 and XIS3) with bursts removed folded on the derived orbital period of $2.928 \mathrm{~h}$ using 64 phasebins per cycle: upper panel: in the band $1.0-4.0 \mathrm{keV}$; middle panel: in the band $4.0-12.0 \mathrm{keV}$. The lower panel is the hardness ratio formed by dividing the lightcurves in the higher and lower energy bands.

in which the time of each dip minimum obtained was plotted against cycle number and these data fitted. In the present observation, the dips consisted of a first minimum followed by a second minimum within the envelope of the full dip, so the lowest point in the second part was used, there being 8 dips in which the time of this was well-defined. This procedure produced a period of $2.928 \pm 0.002 \mathrm{~h}$. This is in good agreement with the previous determinations of $2.938 \pm 0.020 \mathrm{~h}$ from BeppoSAX (Bałucińska-Church et al. 1999) and from Exosat of $2.932 \pm 0.005 \mathrm{~h}$ (Parmar et al. 1989). The data could then be folded on this period and in Fig. 3 we show the XIS lightcurves in two bands: $1.0-4.0 \mathrm{keV}$ and $4.0-12.0 \mathrm{keV}$ and the hardness ratio formed by dividing these. The lightcurve was folded at the epoch of 54109.577080 MJD, which is the time of the first deep dip. Dipping is 55\% deep in the lower energy band and 36\% deep in the higher energy band in the folded light curve. However, folding introduces some smoothing and in the normal lightcurve 
(Fig. 1) dipping is of the order of $65 \%$ deep. The hardness ratio in Fig. 2 shows that lower energies are removed in dipping leading to the observed hardening of the spectrum, which is quite typical for the source.

Similarly, PIN lightcurves extracted in various bands were examined and weak dipping with a depth of $10 \% \pm 6 \%$ can be detected in a folded lightcurve at energies up to $20 \mathrm{keV}$. At higher energies dipping was insignificant.

\subsection{The non-dip non-burst spectrum}

A non-dip, non-burst spectrum was accumulated from the whole observation by firstly noting the times of the start and end of bursts and removing the bursts by selecting on time. To remove dipping intervals and ensure that the shoulders of dipping were completely removed a selection on phase was made, phases 0.28 to +0.28 were removed where the zero of phase was defined as the deepest point of dipping. A spectrum was selected from each of the three XIS detectors; the spectrum in each detector has an exposure time of about $34 \mathrm{ks}$ giving about 84000 counts in each detector (a count rate of 2.5 count s$^{-1}$ ). A background spectrum was accumulated with the same selections for each of the XIS instruments. A response file was generated using the FTOOL XISRMFGEN and a corresponding auxiliary response file also generated for each detector, using the latest calibration database of 18th April, 2008. The ASCA ftool ADDASCASPEC was then used to add the three spectra, the three corresponding background files, the three auxiliary response (arf) files and the three response matrix (rmf) files.

The PIN spectrum was produced using the same GTI files as used to select the XIS data. A burst-like event was noted in the background subtracted PIN lightcurve which occurred $\sim 900 \mathrm{~s}$ before burst No. 2 and lasted $\sim 18 \mathrm{~s}$. No increase of the count rate was detected in any of the three XIS instruments at this time. However, the time interval covering this event was removed from the selection of the PIN persistent emission spectrum. A background spectrum was extracted from the non X-ray background events file provided for the observation, using the same GTI as above. The cosmic X-ray background was added as a term to the spectral model, with photon index $\Gamma=1.29$, a high energy cutoff at $40 \mathrm{keV}$ and normalization of $8.8 \times 10^{-4} \mathrm{ph} \mathrm{cm}^{-2} \mathrm{~s}^{-1} \mathrm{keV}^{-1}$ as required for the observation with the source at the XIS aimpoint.

Deadtime correction of the source spectra was performed using HXDDTCOR. However, this ftool works correctly only for good time intervals longer than 128 seconds. Our PIN spectrum which was selected on orbital phase and for removal of bursts had a tendency of having GTIs in quite short blocks of time, e.g. $16 \mathrm{~s}$. This problems was solved using the standard technique for correcting PIN lightcurves, i.e. by making a lightcurve with $128 \mathrm{~s}$ binning, selecting parts of this with the GTIs required and deadtime corrected by extracting from the "pseudo event file" provided. Systematic errors of 3\% were added to all PIN spectra; no errors were added to XIS as is normal practice.

The XIS spectrum was fitted simultaneously with the PIN spectrum, parameter values being chained between the two. A multiplying factor was included in the model to allow for uncertainty in the relative instrument calibrations and was set to unity for the XIS and free for PIN as is usual practice. Channels below $0.8 \mathrm{keV}$ and above $12 \mathrm{keV}$ in the XIS were ignored where the instrument response is not so well-determined; grouping to a minimum of 100 counts per channel was applied. In the PIN channels between 12 and $70 \mathrm{keV}$ were used, the instrument response at $10-12 \mathrm{keV}$ being less certain and above $70 \mathrm{keV}$ the background was becoming large.

A number of models were tried. Firstly, a cut-off power law gave an acceptable fit with $\chi^{2} /$ d.o.f. $=1415 / 1633$. However, since the 1990s it has generally been accepted that LMXB require a non-thermal and a thermal emission component as published evidence is very strong. White et al. (1988) demonstrated the dominance of the Comptonized emission. Curvature in the continuum spectra at a few $\mathrm{keV}$ cannot be explained as a Comptonization high energy cut-off and requires the presence of a blackbody (Church \& Balucinska-Church 2001), and as said in Sect. 1, the explanation of rapid variability in the dip sources in dipping supports the presence of neutron star blackbody emission. Two-component models are clearly needed to fit the most luminous LMXB (e.g. Schulz et al. 1989; Hasinger et al. 1990) and fitting two-component models became general (e.g. Barret et al. 2000). Consequently, we next added a simple blackbody to the spectral model although in faint sources like XB 1323-619 the thermal component is weak. This two-component continuum model consisting of an absorbed blackbody and cut-off power law $(\mathrm{AG} *(\mathrm{CUT}+\mathrm{BB}))$ gave a significantly better fit for which an F-test showed that it was better with $99.9 \%$ probability. Moreover, the normalization of the blackbody term was found to be significant at $2.6 \sigma$, so from this point we will assume the twocomponent model. However, residuals were present indicating possible emission and several absorption features. Because of the decreased luminosity of the source compared with that at the time of the $X M M$ observation, less line features were positively detected. There was some indication of a weak, broad emission line at $\sim 6.4 \mathrm{keV}$; however as discussed below, we regard detection of this as only tentative. The residuals also indicated an absorption feature at $\sim 6.7 \mathrm{keV}$ and possible absorption features at $\sim 6.6$ and $\sim 6.9 \mathrm{keV}$ as shown in Fig. 5. Finally, a trace of a possible absorption at $\sim 9 \mathrm{keV}$ can be seen in Fig. 5 .

Before adding lines to the spectral model we re-fitted the continuum model excluding the energy range containing the lines $6.5-8.0 \mathrm{keV}$ to prevent the lines slightly distorting the continuum fit. The continuum parameters were then frozen and lines added. The width of the emission line was fixed (at $0.02 \mathrm{keV}$ ) to prevent the line absorbing neighbouring continuum. Although the emission feature at $\sim 6.4 \mathrm{keV}$ had an equivalent width $E W$ of $17 \pm 7 \mathrm{eV}$, we do not include it in Table 2 showing the fit results as we regard the detection as tentative as the appearance of the line in the residuals is not totally convincing. Initially a single absorption line was added with $\sigma$ fixed at $0.01 \mathrm{keV}$ as all the absorption features appeared narrow. This fitting resulted in a line at 6.6(7) $\pm 0.05 \mathrm{keV}$ being detected with $E W=12 \pm 7 \mathrm{eV}$, and these results are shown in Table 2. This line can be identified with Fe XXV and so represents absorption by highly ionized material which could be located in the ADC.

However, the residuals indicate there are probably at least three weak absorption lines and we attempted to fit the spectrum with three lines. In this case, it was not possible to determine the energies by free fitting so these were fixed at values obtained by inspection of the residuals, i.e. $6.59,6.74 \mathrm{keV}$ and $6.90 \mathrm{keV}$. It was not possible to get a sensible fit to the residual feature at $6.9 \mathrm{keV}$, i.e. the normalization was zero. The other two lines at $\sim 6.59$ and $\sim 6.74 \mathrm{keV}$ were found to have $E W \mathrm{~s}$ of $-6.8 \pm 6.8 \mathrm{eV}$ and $-6.4_{-7.4}^{+6.4} \mathrm{eV}$ and correspond to marginal $1 \sigma$ detections, and for this reason we do not show them in Table 2. As said above, the energies are uncertain and we should be careful not to over-interpret them. The feature at $6.59 \mathrm{keV}$ may correspond to a less ionized absorber, that is, to an $\mathrm{Fe} \mathrm{K}$ transition above about Fe XVII. As we see Fe XXV (Table 2) and also 
Table 2. Best simultaneous fit to the non-dip and dip XIS + PIN spectra.

\begin{tabular}{|c|c|c|}
\hline Model Parameters & Non-dip & 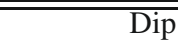 \\
\hline$N_{\mathrm{H}}\left(10^{22}\right.$ atom $\left.\mathrm{cm}^{-2}\right)$ & $3.2 \pm 0.1$ & $3.2 \pm 0.1$ \\
\hline $\begin{array}{l}\text { CUTOFF POWER LAW } \\
\Delta N_{\mathrm{H}}\left(10^{22} \text { atom } \mathrm{cm}^{-2}\right) \\
\text { Covering fraction } f \\
\text { photon index } \Gamma \\
\text { cut-off energy }(\mathrm{keV}) \\
\text { normalization }\left(10^{-2} \mathrm{ph} \mathrm{cm}^{-2} \mathrm{~s}^{-1}\right)\end{array}$ & $\begin{array}{r}0.0 \\
0.0 \\
1.67_{-0.03}^{+0.10} \\
85_{-35}^{+77} \\
2.4_{-0.3}^{+0.9}\end{array}$ & $\begin{array}{r}22.2 \pm 0.8 \\
0.63 \pm 0.02\end{array}$ \\
\hline $\begin{array}{l}\text { BLACKBODY } \\
\Delta N_{\mathrm{H}}\left(10^{22} \text { atom } \mathrm{cm}^{-2}\right) \\
\text { temperature } k T_{\mathrm{BB}}(\mathrm{keV})^{\text {normalization }\left(10^{-5} \mathrm{erg} \mathrm{s}^{-1} \text { at } 10 \mathrm{kpc}\right)}\end{array}$ & $\begin{array}{r}0.0 \\
1.35 \pm 0.36 \\
8.3 \pm 5.1\end{array}$ & $>400$ \\
\hline $\begin{array}{l}\text { ABSORPTION LINE } \\
\text { energy } E(\mathrm{keV}) \\
\text { width } \sigma(\mathrm{keV}) \\
\text { normalization }\left(10^{-5} \mathrm{ph} \mathrm{cm}^{-2} \mathrm{~s}^{-1}\right) \\
\text { equivalent width } E W(\mathrm{eV})\end{array}$ & & $\begin{array}{r}6.52 \pm 0.10 \\
0.01 \text { (frozen) } \\
-1.0 \pm 0.8 \\
-18 \pm 15\end{array}$ \\
\hline $\begin{array}{l}\text { ABSORPTION LINE } \\
\text { energy } E(\mathrm{keV}) \\
\text { width } \sigma(\mathrm{keV}) \\
\text { normalization }\left(10^{-5} \mathrm{ph} \mathrm{cm}^{-2} \mathrm{~s}^{-1}\right) \\
\text { equivalent width } E W(\mathrm{eV})\end{array}$ & $\begin{array}{r}6.67 \pm 0.05 \\
0.01 \text { (frozen) } \\
-1.24 \pm 0.71 \\
-12 \pm 7\end{array}$ & $\begin{array}{r}6.68_{-0.02}^{+0.18} \\
0.01 \text { (frozen) } \\
-0.9_{-0.9}^{+0.8} \\
-15 \pm 15\end{array}$ \\
\hline $\begin{array}{l}\text { ABSORPTION LINE } \\
\text { energy } E(\mathrm{keV}) \\
\text { width } \sigma(\mathrm{keV}) \\
\text { normalization }\left(10^{-5} \mathrm{ph} \mathrm{cm}^{-2} \mathrm{~s}^{-1}\right) \\
\text { equivalent width } E W(\mathrm{eV})\end{array}$ & & $\begin{array}{r}6.94 \pm 0.14 \\
0.01 \text { (frozen) } \\
-1.5 \pm 1.4 \\
-26 \pm 25\end{array}$ \\
\hline $\begin{array}{l}\text { ABSORPTION LINE } \\
\text { energy } E(\mathrm{keV}) \\
\text { width } \sigma(\mathrm{keV}) \\
\text { normalization }\left(10^{-5} \mathrm{ph} \mathrm{cm}^{-2} \mathrm{~s}^{-1}\right) \\
\text { equivalent width } E W(\mathrm{eV})\end{array}$ & & $\begin{array}{r}7.6 \pm 0.2 \\
0.01 \text { (frozen) } \\
-2.8 \pm 2.1 \\
-68 \pm 52\end{array}$ \\
\hline dip/non-dip intensity shift & & $0.79 \pm 0.04$ \\
\hline PIN normalization factor: & $1.12 \pm 0.07$ & 1.12 (frozen) \\
\hline $\begin{array}{l}\chi^{2} / \text { d.o.f. } \\
\chi_{\mathrm{r}}^{2}\end{array}$ & $\begin{array}{r}1381 / 1634 \\
0.85\end{array}$ & $\begin{array}{r}140 / 263 \\
0.53\end{array}$ \\
\hline
\end{tabular}

90\% confidence errors are given for all quantities except for the weaker 6.52 and $6.68 \mathrm{keV}$ lines where $67 \%$ confidence errors are shown for all parameters.

absorption by neutral absorber in dipping, absorption by an intermediate ionization state may be quite possible. In Fig. 4 we show the folded data and residuals for the full band of the XIS and PIN instruments, while in Fig. 5 (upper panel) we show the absorption features in the residuals over the band $5-10 \mathrm{keV}$ before lines were added to the spectral model.

In the best-fit solution, the best-fit power law photon in$\operatorname{dex} \Gamma$ of $1.67_{-0.03}^{+0.10}$ is very similar to that we obtained in the $X M M$ observation of $1.68 \pm 0.08$ (Church et al. 2005) and is physically reasonable; the cut-off energy of $85 \mathrm{keV}$ is high as expected in a low luminosity source (a value of $44.1_{-4.4}^{+5.1}$ was obtained in the broadband $S A X$ observation (BałucińskaChurch et al. 1999). In the analysis of the XMM observation, absorption at $6.70 \pm 0.03 \mathrm{keV}$ was seen and $6.98 \pm 0.04 \mathrm{keV}$, and broad emission at about $6.8 \mathrm{keV}$ (Church et al. 2005).
Barnard et al. (2001) detected an emission line at $6.43 \pm 0.21 \mathrm{keV}$ in a RXTE observation of XB 1323-619.

\subsection{The broadband deep dip spectrum}

It is, of course, of interest to investigate the spectrum of deep dipping, to use the broadband capacity of Suzaku to reveal the energy dependence of dipping, and test whether we can model the spectral evolution. In particular, we apply the "progressive covering" model for dipping (Church et al. 1997) in which the extended absorbing bulge on the outer accretion disk progressively overlaps the extended ADC source of Comptonized emission, this model having previously provided a very good description of spectral evolution in dipping in all dipping LMXB (Church et al. 1997). We will also test the extent to which 
Suzaku XIS+PIN

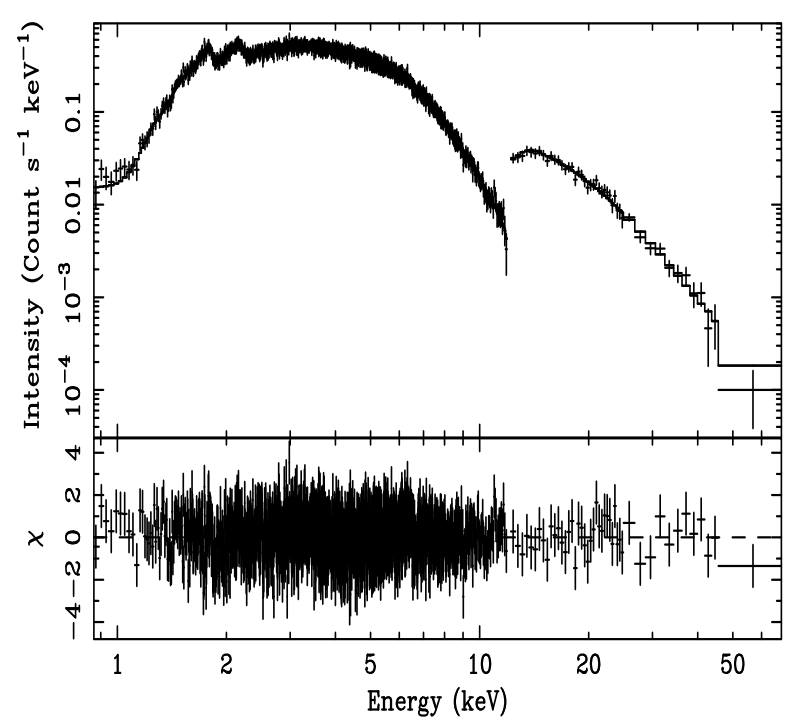

Fig. 4. Best fit to non-dip, non-burst spectrum of the three XIS detectors fitted simultaneously with the PIN detector; for details of the line features detected see Fig. 5 and the text.
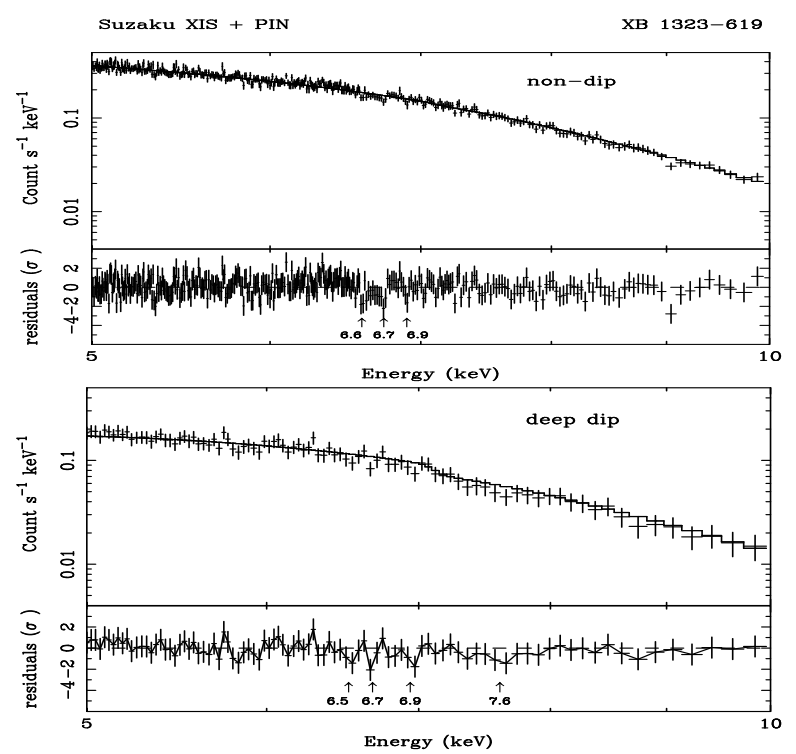

Fig. 5. Upper panel: persistent emission spectrum and model fit in the band $5-10 \mathrm{keV}$ before addition of any line features showing several absorption features; lower panel: the corresponding residuals for the deep dip spectrum.

dipping can be described as photoelectric absorption, and the degree to which electron scattering may take place, given the suggestion by Boirin et al. (2005) based on analysis of the XMM observation of XB 1323-619 that spectral evolution in dipping in all the LMXB dipping sources might be explained by photoelectric absorption plus electron scattering in a medium of varying ionization parameter.

Dip data were selected from the deepest part of dipping where dipping is $\sim 65 \%$ deep, by selecting for intensities below 1.2 count $\mathrm{s}^{-1}$ in XISO and applying the resulting GTI to all instruments. In addition, we removed bursts and selected for phases betweem -0.16 and +0.09 . Firstly, we fitted the XIS and PIN dip spectra with a model of the form $\mathrm{AG} *\left(\mathrm{PCF} * \mathrm{CUT}+\mathrm{AG}_{1} * \mathrm{BB}\right)$ i.e. applying the covering factor PCF to the cut-off power law

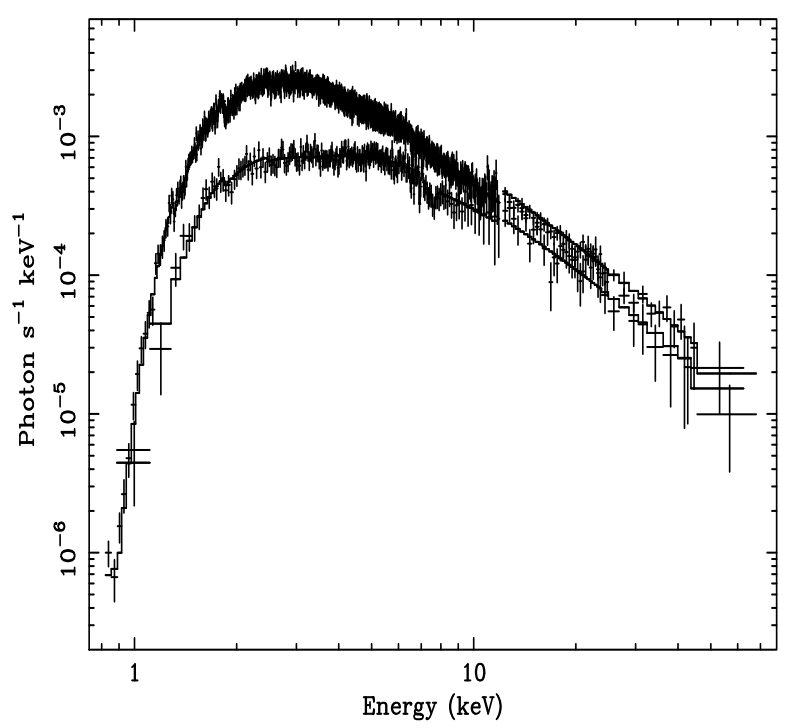

Fig. 6. Comparison of non-dip and deep dip spectra from best fits to XIS and PIN simultaneously in each. For clarity individual model components are not shown to demonstrate the energy-dependent nature of the dipping.

modelling the extended emission of the ADC, while the pointlike blackbody is subject to a column density $\mathrm{AG}_{1}$ in addition to the Galactic column density AG, frozen at the non-dip value. An assumption of this model is that emission parameters cannot vary in dipping, only absorption parameters and so all of the continuum emission parameters of the blackbody and cut-off power law were frozen at their non-dip values.

Acceptable fits were obtained, with $\chi^{2} /$ d.o.f. $\sim 1$ but with clear residuals indicating the presence of absorption features. The emission line seen at $\sim 6.4 \mathrm{keV}$ in non-dip emission could not be seen in the residuals, and attempts to fit such a line resulted in a value of zero for the line normalization. Consequently no emission line was included in dip fitting. Inspection of the residuals revealed absorption lines at $\sim 6.5, \sim 6.7, \sim 6.9$ and $\sim 7.6 \mathrm{keV}$. Corresponding lines were added to the spectral model. The line at $7.6 \mathrm{keV}$ appeared broader than the other lines in the residuals with a full width at zero height of $\sim 0.6 \mathrm{keV}$ and so its width $\sigma$ was initially set at a corresponding $0.15 \mathrm{keV}$ in the fitting, while the widths $\sigma$ of the first three lines were frozen at a value appropriate to narrow lines $(0.01 \mathrm{keV})$.

A formally acceptable fit could be obtained in this way. However, a comparison of the non-dip and deep dip spectra shown in Fig. 6 shows a vertically downwards shift in the dip spectrum, i.e. an energy-independent decrease of intensity. We investigated this, and determined the level of any energyindependent change during dipping by adding a multiplying factor to the spectral model, this factor affecting both cut-off power law and blackbody terms. All emission parameters were fixed at the non-dip values and the XIS-PIN normalization factor was fixed at the non-dip value of 1.12. There was a substantial improvement in the quality of fit with $\chi^{2} /$ d.o.f. becoming $140 / 263$ compared with the value without the vertical shift factor (164/263). Best-fit values for the final fit to the dip spectrum are shown in Table 2. The dipping was well explained by an increase in column density $\left(\Delta N_{\mathrm{H}}\right.$ of at least $400 \times 10^{22}$ atom cm $\mathrm{cm}^{-2}$ and covering of $63 \%$ of the Comptonized emission by absorber, with a smaller increase of $N_{\mathrm{H}}$ of $22 \times 10^{22}$ atom $\mathrm{cm}^{-2}$. This suggests that the blackbody is covered by central, denser regions of 
the absorbing bulge whereas the Comptonized emission is overlapped by lower density regions on average. Note that the best fit requires an unabsorbed fraction of $37 \%$, i.e. part of the spectrum not overlapped is unabsorbed. In addition to the neutral absorption, the dip spectrum requires an energy-independent decrease by a factor of $0.79 \pm 0.04$. It was found that the covering factor $f$ for the dip spectrum of $0.63 \pm 0.02$ agreed well with the depth of dipping in the lightcurve of $\sim 65 \%$. Figure 6 shows that below $\sim 20 \mathrm{keV}$ dipping is strongly energy-dependent as modelled by neutral absorber in our fitting. Above $20 \mathrm{keV}$ the small, approximately energy-independent vertical shift can be seen.

Table 2 also shows the best-fit line energies, all obtained by free fitting. The lines at $6.94 \mathrm{keV}$ and $7.6 \mathrm{keV}$ were detected at the 3- $\sigma$ level or higher, however, the other two lines were weaker and were only detected at $1-\sigma$ and so we show these lines in the table with 1- $\sigma$ errors. Figure 5 (lower panel) shows the absorption features using the best-fit continuum model with the lines removed from the spectral model. The line at $\sim 6.5 \mathrm{keV}$ apparently corresponds to the weak feature seen in the non-dip spectrum which we suggested was due to a less ionized Fe state. Possibly more than a single line is present, i.e. a forest of lines from $\sim$ Fe XVII onwards. There appears no doubt that the line at $6.68 \mathrm{keV}$ is Fe XXV as seen in non-dip. The equivalent widths of these lines are greater than in non-dip; however, this may be due to the continuum decrease ( $E W=$ line flux/continuum intensity). But because of the large uncertainties in $E W$ values, we can only say that the increase in $E W$ is consistent with the change in continuum. Indeed, it would not be expected that the bulge in the outer disk would cause increased absorption in high ionization states. The line at $6.94 \mathrm{keV}$, also seen in the observation with $X M M$ (Church et al. 2005), can be identified as Fe XXVI $\mathrm{K}_{\alpha}$, and the line at $7.6 \mathrm{keV}$ can be Ni XXVI.

As a check, we also examined the energy-independent spectral change in the PIN spectrum alone, so as to be independent of XIS-PIN normalization using the energy band 20-70 keV where the effects of photoelectric absorption are small. We carried out spectral fitting in this band using a cut-off power law (as the blackbody flux is negligible) fitting the non-dip spectrum, and then fixing the power law index and cut-off energy in fitting the dip spectrum. The normalization decreased by $24 \pm 3 \%$, agreeing with the value of $21 \pm 4 \%$ obtained from simultaneous fitting of XIS and PIN; however this result may be biassed by points at $20 \mathrm{keV}$ where absorption makes a small contribution and we conclude that our best value for the degree by which electron scattering reduces the intensity is the factor shown in Table 3 from fitting both instruments simultaneously.

\section{Discussion}

Our spectral fitting results show that dipping consists of a large increase in photoelectric absorption for the Comptonized emission of the ADC, the intrinsic column density increasing from zero to $22 \times 10^{22}$ atom $\mathrm{cm}^{-2}$ in deep dipping, this being consistent with covering of an extended ADC source by an extended absorbing bulge in the outer disk as previously found for the dipping sources in general, and for XB 1323-619 in the observations with BeppoSAX (Bałucińska-Church et al. 1999), with RXTE (Barnard et al. (2001) and XMM (Church et al. 2005). The neutron star blackbody is subject to a high column density which quickly removes this component in dipping as expected for a point-source and consistent with the source being covered by the highest column density part of the bulge in the outer disk, rather than an integration across the whole bulge as for the extended Comptonized emission.
An energy-independent shift can be seen at energies above $20 \mathrm{keV}$ where photoelectric absorption is negligible indicating a degree of electron scattering, the shift being $21 \pm 4 \%$. A decrease of $10 \pm 10 \%$ was found in our observation of the source with BeppoSAX in the band 20-50 keV (Bałucińska-Church et al. 1999). We can show that the actual high energy shift is consistent with the degree of electron scattering expected for the electrons present in the bulge in the outer disk. At an energy of $5 \mathrm{keV}$, the photoelectric absorption cross section $\sigma_{\mathrm{PE}}$ is about 10 times the Thomson cross section $\sigma_{\mathrm{T}}$ and the column density for the

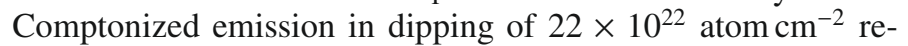
duces the intensity by a factor $\exp -N_{\mathrm{H}} \sigma_{\mathrm{PE}} \sim 0.46$ for the $63 \%$ of the emission that is covered, so that the overall factor is $0.46 \times 0.63+0.37$, i.e. a decrease of about $50 \%$ at $5 \mathrm{keV}$ close to the actual decrease as seen in Fig. 6.

At $20 \mathrm{keV}, \sigma_{\mathrm{PE}}$ is 10 times less than $\sigma_{\mathrm{T}}$ and at higher energies becomes negligible. The electron density in the absorbing bulge in the outer disk will be between 1.0 and 1.2 times the ion density depending on the exact ionization state, because of multiple electrons released in ionization of the metals so that for an electron column density $N_{\mathrm{e}}$ of $\sim 22 \times 10^{22}$ atom $\mathrm{cm}^{-2}$ electron scattering reduces the X-ray intensity of the covered part of the extended emission by a factor 0.86 , so that the overall factor is $0.86 \times 0.63+0.37$, i.e. 0.90 , a $10 \%$ reduction in intensity, somewhat less than our measured decrease of $21 \%$, although the errors are substantial. Our spectral fitting was carried out using the cross sections for neutral absorber. We show below that the ionization parameter $\xi=L / n r^{2}$, where $L$ is the luminosity, $n$ is the plasma density, and $r$ the radial position, can be no more than 50 in the outer disk because of the large distance from the neutron star. For $\xi$ smaller than $\sim 15$, the degree of ionization in the bulge will be low, i.e. there will be a large neutral atom component. For $\xi$ between 15-50, only a low ionization state is achieved; for example, the oxygen ion may reach a state O II-O VI, but not the higher states O VIII or O IX (Kallman \& McCray 1982). The absorption cross sections are reduced from the neutral absorber values and in oxygen, $\sigma_{\mathrm{PE}}$ is reduced by up to a factor of $\sim 2$ for O V/O VI (Verner et al. 1996). Thus spectral fitting would require a column density up to two times higher than for neutral absorber so that $N_{\mathrm{e}}$ would also be higher and the reduction in intensity by electron scattering would rise to $20 \%$. At higher energies, e.g. 20-70 keV, the Klein-Nishina formula shows that the mean Thomson cross section in this band decreases by $12 \%$, but this has little effect on the reduction in intensity so that, the expected decrease would again be $20 \%$.

Thus application of the progressive covering absorption model to the Extended ADC LMXB emission model provides a very good description of the spectral evolution in dipping, including both the effects of photoelectric absorption and electron scattering in the bulge in the outer disk. Assuming that the bulge has a radial extent of about $10 \%$ of the disk radius $\left(\sim 3.5 \times 10^{10} \mathrm{~cm}\right)$ and using the measured column density in dipping for the extended emitter (Table 3 ) we can estimate an average density in the bulge of $\sim 7 \times 10^{13} \mathrm{~cm}^{-3}$. From this it follows that the ionization parameter $\xi$ cannot be larger than $\sim 50$, so that the matter is in a relatively low ionization state $(\xi \sim 1000$ is needed for a high ionization state). If our measured column densities are too low because we employ a neutral absorber model, $\xi$ would be even smaller.

Suggestions have been made by Díaz-Trigo et al. (2006) and Boirin et al. (2005) that dipping in all of the dipping LMXB, i.e. the large decreases in continuum flux, can be explained not in terms of absorption in the bulge in the outer disk but in an ionized absorber with high ionization state $(\log \xi>3)$ 
suggesting that the absorber is located much closer to the neutron star, although they do not specify the radial position of the absorber. It is proposed from this fitting that a progressive covering description of dipping is not needed so that there is no need for an extended Comptonizing region. However, the questions of why the bulge in the outer disk does not cause strong absorption, and what azimuthal structure causes dipping in their model are not addressed.

Detailed recent work does reveal the presence of highly ionized line features in the dipping LMXB. For example, Jimenez-Garate et al. (2003) investigated such features in XBT 0748-676 and concluded that dipping was due to neutral absorber in the outer disk plus some contribution from ionized absorber also located in the outer disk but in layers above the disk. Parmar et al. (2002) report detection of broad emission and narrow absorption features in X 1624-490 using XMM-Newton. Lines present in dipping indicated that the additional absorbing material had lower ionization state than that producing the narrow absorption features seen in persistent emission.

However, the idea of a lower ionization state in dipping was taken by Díaz-Trigo et al. (2006) to mean that a single region of high but changing ionization state could describe both non-dip and dip spectra, without reference to the bulge in the outer disk. Spectral evolution in dipping in several sources was modelled using a model $\mathrm{ABS} * \mathrm{XABS} *(\mathrm{BB}+\mathrm{PL})$ where $\mathrm{ABS}$ is neutral absorber and XABS is ionized absorber. This model had free parameters $\xi$, ionized absorber column density $N_{\text {ion }}$ and a line width $\sigma$. The main parameter changes in dipping were a decrease of $\log \xi$ from 3.5 typically to 3.0 , an increase of $N_{\text {ion }}$ by ten times, e.g. from $4 \times 10^{22}$ to $40 \times 10^{22}$ atom $\mathrm{cm}^{-2}$ and a small increase in the column density of neutral absorber, e.g. typically by up to a

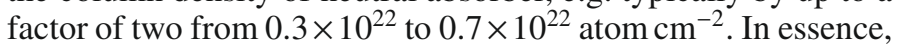
this fitting still explains dipping as photoelectric absorption but in a region where the net effective cross section of the mixture of elements is reduced as the metals are in higher ionization states. The continuum change in dipping can be fitted, but the high $\xi$ has the consequence that the absorber has to be located much closer to the neutron star than the bulge.

Thus Díaz-Trigo et al. (2006) based on this spectral fitting, and Boirin et al. (2005), conclude that dipping can be explained without progressive covering of an extended emission region as applied extensively to the dipping sources, e.g. by Church et al. (1997). They further suggest that an extended ADC is not required in their fitting, which is the basis of the Extended ADC emission model for LMXB in general (Church \& Bałucińska-Church 2004) for which substantial evidence exists. Firstly, fitting non-dip and dip spectra in any dipping LMXB shows that the majority of the X-ray flux, i.e. the non-thermal emission, is removed gradually and systematically proving that the emitter is extended (e.g. Church et al. 1997). The size of the Comptonization emission site has been measured by dip ingress timing for all of the dipping LMXB (Church \& BałucińskaChurch 2004) showing that this emitter is indeed very large, having a radial extent typically $50000 \mathrm{~km}$ or $5-50 \%$ of the accretion disk radius. Strong independent evidence for an extended hot ADC is given by Schulz et al. (2009) who detect a variety of broad emission lines of highly ionized species including NeX $L_{\alpha}, \operatorname{Mg}$ XII $L_{\alpha}$, Si XIV $L_{\alpha}$, S XVI $L_{\alpha}$, Fe XXIV, Fe XXV and Fe XXVI in Chandra grating spectra of Cygnus X2 . The emissivities indicate $\log \xi>3$, and the radial positions of the emitters were found to be between 20000 and $110000 \mathrm{~km}$ from the neutron star. It was thus concluded that these lines originate in a hot, extended ADC and the radial distances are in excellent agreement with the results of dip ingress timing.
Schulz et al. (2008) in a study of Cir X-1 similarly found a large number of emission line features rich in $\mathrm{H}$-like and He-like features of high Z-elements such as Si, S, Ar and Ca. The spectra could be modelled as emission from a hot $\left(T \sim 10^{7} \mathrm{~K}\right)$ photoionized plasma with $\xi=1000$, located at a radial distance $r \sim 10000 \mathrm{~km}$ from the neutron star, i.e. a hot extended accretion disk corona.

Given the now apparent overwhelming evidence for the existence of extended ADCs, it seems necessary to include such an extended emitter in any model fitting (i.e. by a progressive covering term), which is quite in contrast to simply using an ionized absorber as done, for example, in Diaz-Trigo et al. (2006). In addition, there are a number of elements lacking in the model which appear difficult to explain in terms of the model, as follows, which are not a problem for the standard explanation of dipping. It is not specified at what radial position the ionized absorber exists, nor is it suggested what causes the azimuthal structure required in the absorber to cause dipping in a particular range of orbital phases. It is difficult to see why there should be any azimuthal structure in an inner region where $\xi$ is high, especially since such structure would have to be similar to the known structure in the outer disk, i.e. the bulge. Finally the model does not explain why the bulge should have no absorbing effect contrary to accepted ideas. Contrasted with this should be the existing explanations of dipping as progressive covering of the neutron star and an extended ADC by the extended absorbing bulge, which is physically reasonable, with substantial evidence for it.

From the continuum changes, we turn to the line features detected in the Suzaku observation (Table 2). A possible emission line consistent with $6.4 \mathrm{keV}$ was seen in the Suzaku non-dip spectrum which was absent in the dip spectra. A similar feature had been seen in the 1997 RXTE spectrum at $6.43 \pm 0.21 \mathrm{keV}$ (Barnard et al. 2001) although the line was not found in the $2003 X M M$ observation when the source was much brighter. Absorption lines were also seen: in the non-dip spectrum the only line we can be confident of was the $6.67 \mathrm{keV} \mathrm{Fe} \mathrm{XXV} \mathrm{line.}$ In dipping, four absorption lines were seen as discussed previously. The general presence of these medium to high ionization features are consistent with formation in a hot ADC and this hot extended ADC is, of course, a major element of the "Extended ADC" continuum emission model (Church \& Bałucińska-Church 2004). Other authors, in particular Schulz et al. $(2008,2009)$ have presented strong evidence that similar lines originate in an extended, hot ADC.

In the Suzaku observation of XB 1323-619 the luminosity was at a low level in contrast with the highest luminosity exhibited by the source in the 2003 XMM observation, after which the luminosity was reduced by about a factor of two in the RXTE observation of 2003, and fell further by almost another factor of two in the present observation. The X-ray burst rate has also decreased substantially to about the value it had in the Exosat observation, as shown in Fig. 2. The present burst rate supports the idea that the burst rate follows a definite burst rate-luminosity relation which has not changed over 20 years. We found in the present observation that the typical long burst decays having two time constants indicative of burning by the rp-process. Such behaviour was found previously in the source prior to the $X M M$ observation. However, in the XMM observation it was found that the long tails were not seen in bursts indicating less rp-burning (Bałucińska-Church et al. 2008), a possible reason being incomplete burning of $\mathrm{H}$ to $\mathrm{He}$ between bursts at the higher luminosity and mass accretion rate. The return of the burst properties of the source to those determined for the Exosat, RXTE and BeppoSAX observations prior to the $X M M$ observation support the idea that 
the burst behaviour is determined primarily by the mass accretion rate.

The evidence is that the luminosity measured in the Exosat and Suzaku observations is a lower limit for the source. Between 1985 and 2003, the luminosity increased by a factor of about 4, implying that the mass accretion rate increased by that factor. We cannot say whether this behaviour is periodic or not, however, the observed gradual increase over an 18 year period followed by a much faster decrease in luminosity over 4 years does not suggest a periodic change, and further observations will reveal whether the luminosity will increase as it did from 1985 onwards. If the source were periodic, it could possibly be a triple system, in which case the third body would need to have a period of the order of 30 days.

Acknowledgements. We are grateful to the referee for his very helpful comments. This work was supported in part by the Polish Ministry of Higher Education and Science grants KBN-1528/P03/2003/25 and 3946/B/H03/2008/34, and by PPARC grant PP/C501884/1. We thank P. J. Humphrey for providing additional analysis tools

\section{References}

Angelini, L., Church, M. J., Parmar, A. N., Bałucińska-Church, M., \& Mineo, T. 1998, A\&A, 339, L41

Bałucińska-Church, M., Church, M. J., Oosterbroek, T., et al. 1999, A\&A, 349, 495

Bałucińska-Church, M., Humphrey, P. J., Church, M. J., \& Parmar, A. N. 2000, A\&A, 360, 583

Bałucińska-Church, M., Reed, D., \& Church, M. J. 2008, Chin. J. Astron. Astrophys., 8, 319

Barnard, R., Bałucińska-Church, M., Smale, A. P., \& Church, M. J. 2001, A\&A, 380,494

Barret, D., Olive, J. F., Boirin, L., et al. 2000, ApJ, 533, 329
Boirin, L., Méndez, M., Díaz Trigo, M., Parmar, A. N., \& Kaastra, J. S. 2005, A\&A, 436, 195

Church, M. J., \& Bałucińska-Church, M. 2001, A\&A, 369, 915

Church, M. J., \& Bałucińska-Church, M. 2004, MNRAS, 348, 955

Church, M. J., Mitsuda, K., Dotani, T., et al. 1997, ApJ, 491, 388

Church, M. J., Bałucińska-Church, M., Dotani, T., \& Asai, K. 1998a, ApJ, 504, 516

Church, M. J., Parmar, A. N., Bałucińska-Church, M., et al. 1998b, A\&A, 338, 556

Church, M. J., Reed, D., Dotani, T., Bałucińska-Church, M., \& Smale, A. P. 2005, 359, 1336

Church, M. J., Halai, G. S., \& Bałucińska-Church, M. 2006, A\&A, 460, 233

Cornelisse, R., in't Zand, J. J. M., Verbunt, F., et al. 2003, A\&A, 405, 1033

Díaz-Trigo, M., Parmar, A. N., Boirin, L., Méndez, M., \& Kaastra, J. S. 2006, A\&A, 445, 179

Forman, W., Jones, C., Cominsky, L., et al. 1978, ApJS, 38, 357

Galloway, D. K., Cumming, A., Kuulkers, E., et al. 2004, ApJ, 601, 466

Hasinger, G., van der Klis, M., Ebisawa, K., Dotani, T., \& Mitsuda, K. 1990, A\&A, 235, 147

Jimenez-Garate, M. A., Raymond, J. C., \& Liedahl, D. A. 2002, ApJ, 581, 1297

Jimenez-Garate, M. A., Schulz, N. S., \& Marshall, H. L. 2003, ApJ, 590, 432

Kallman, T. R., \& McCray, R. 1982, ApJS, 50, 263

Langmeier, A., Sztajno, M., Hasinger, G., \& Trümper, J. 1987, ApJ, 323, 288

Parmar, A. N., Gottwald, M., van der Klis, M., \& van Paradijs, J. 1989, ApJ, 338, 1024

Parmar, A. N., Oosterbroek, T., Boirin, L., \& Lumb, D. 2002, A\&A, 386, 910

Schulz, N. S., Hasinger, G., \& Truemper, J. 1989, A\&A, 225, 48

Schulz, N. S., Kallman, T. E., Galloway, D. K., \& Brandt, W. N. 2008, ApJ, 672, 1091

Schulz, N. S., Huenemoreder, D. P., Ji, L., et al. 2009, ApJ, 692, L80

Smale, A. P., Church, M. J., \& Bałucińska-Church, M. 2001, ApJ, 550, 962

Van der Klis, M., Jansen, F., van Paradijs, J., \& Stollman, G. 1985, Space Sci. Rev., 30, 512

Verner, D. A., Ferlan, G. J., Korista, K. T., \& Yakovlev, D. G. 1996, ApJ, 465, 487

Wallace, R. K., \& Woosley, S. E. 1981, APJS, 45, 389

Walter, F. M., Mason, K. O., Clarke, J. T., et al. 1982, ApJ, 253, L67

Warwick, R. S., Marshall, N., Fraser, G. W., et al. 1981, MNRAS, 197, 865

White, N. E., \& Swank, J. H. 1982, ApJ, 253, L61

White, N. E., Stella, L., \& Parmar, A. N. 1988, ApJ, 324, 363 